ARTICLE

Received 17 Dec 2014 | Accepted 19 May 2015 | Published 7 Jul 2015

DOI: $10.1038 /$ ncomms 8545

OPEN

\title{
Genomic modulators of gene expression in human neutrophils
}

Vivek Naranbhai ${ }^{1}$, Benjamin P. Fairfax ${ }^{1}$ Seiko Makino ${ }^{1}$, Peter Humburg${ }^{1}$, Daniel Wong ${ }^{1}$, Esther Ng$^{1}$, Adrian V.S. Hill ${ }^{1} \&$ Julian C. Knight ${ }^{1}$

Neutrophils form the most abundant leukocyte subset and are central to many disease processes. Technical challenges in transcriptomic profiling have prohibited genomic approaches to date. Here we map expression quantitative trait loci (eQTL) in peripheral blood CD16 + neutrophils from 101 healthy European adults. We identify cis-eQTL for 3281 neutrophil-expressed genes including many implicated in neutrophil function, with 450 of these not previously observed in myeloid or lymphoid cells. Paired comparison with monocyte eQTL demonstrates nuanced conditioning of genetic regulation of gene expression by cellular context, which relates to cell-type-specific DNA methylation and histone modifications. Neutrophil eQTL are markedly enriched for trait-associated variants particularly autoimmune, allergy and infectious disease. We further demonstrate how eQTL in PADI4 and NOD2 delineate risk variant function in rheumatoid arthritis, leprosy and Crohn's disease. Taken together, these data help advance understanding of the genetics of gene expression, neutrophil biology and immune-related diseases.

\footnotetext{
${ }^{1}$ Wellcome Trust Centre for Human Genetics, Nuffield Department of Medicine, University of Oxford, Oxford OX3 7BN, UK. Correspondence and requests for materials should be addressed to V.N. (email: vivekn@well.ox.ac.uk) or to B.P.F. (email: bfairfax@well.ox.ac.uk) or to J.C.K. (email:

Julian.knight@ndm.ox.ac.uk).
} 
$\mathrm{V}$ ariation in the human genome is a major regulatory mechanism of gene transcription ${ }^{1}$. Regulatory variants may modulate gene expression of local genes (cis-eQTL, likely acting on the same chromosome) or genes at a distance on non-contiguous chromosomes (trans-eQTL). Quantitative variation in transcription frequently leads to protein variation ${ }^{2}$ leading to phenotypic traits. Although cell-type-specific effects were noted in early studies $^{3}$, ease of sample availability may explain why the largest studies of eQTL are in cell lines ${ }^{4-8}$ or mixed populations of leukocytes from peripheral blood ${ }^{9-12}$. Studies of eQTL in monocytes ${ }^{13-15}$, monocyte-derived dendritic cells $^{16}$, B lymphocytes ${ }^{13}$, regulatory $\mathrm{T}$ cells ${ }^{17}, \mathrm{CD} 4+\mathrm{T}$ cells or bulk $\mathrm{T}$ cells ${ }^{3}$ demonstrate that the effects of a genetic variant on gene expression differ according to cell type and these are further conditioned by cellular activation state and stimulatory environment ${ }^{18-20}$. Similar results comparing tissues support this conclusion ${ }^{21-24}$. Collectively, these studies demonstrate the need for context-specific eQTL mapping in diverse primary populations of cells informative for disease.

Neutrophils make up $40-70 \%$ of the total circulating leukocyte pool and due to their abundance in blood and tissue, they are frequently observed in tissue specimens with minimal blood contamination. There has been one recent study of eQTL in murine neutrophils ${ }^{25}$, but the genetic architecture of gene expression in human neutrophils remains unclear. Challenges in isolating neutrophils ${ }^{26}$ (as opposed bulk granulocytes that paradoxically bias RNA measures towards eosinophils ${ }^{27}$ ) have limited the ability to study these cells leading to attempts at in silico prediction of neutrophil eQTL ${ }^{28}$ from whole-blood data. About $10^{11}$ neutrophils are produced daily in the adult human bone marrow and constitute first-responders in the innate immune response to a variety of infectious and non-infectious insults $^{29}$. Neutrophils are characterized by multilobed nuclei and an abundance of primary, secondary and tertiary solubledefence-mediator-filled granules. Although neutrophils are classically thought to be short-lived cells, they play roles in acute and chronic inflammation and can migrate into tissues or return to the blood compartment and survive $e^{30}$. Neutrophils have extensive crosstalk with each of the major blood cell subsets (megakaryocyte, myeloid and lymphoid) ${ }^{31-34}$ further magnifying their function in health and disease. Neutrophils are thus central to orchestrating immune responses and understanding the regulation of gene expression in neutrophils may, we hypothesized, offer insights into disease biology.

We identified cis-acting genetic modulators of gene expression in neutrophils for more than 3,000 genes, including dozens involved in the development, migration and function of neutrophils. Comparison with other cell types and paired analysis of neutrophil and monocyte eQTL demonstrates how cell-type modifies the effect of eQTL. Moreover, cell-type-specific epigenetic data help resolve the mechanisms of cell-type constraint of eQTL and fine-map causal variants. We show how variants that affect gene expression are implicated in hundreds of common diseases. We interrogate two such associations. First, we show how integration of neutrophil and monocyte eQTL and epigenetic data with genome-wide association study (GWAS) data implicates PADI4 expression in neutrophils in rheumatoid arthritis susceptibility. Second, we show how a single-nucleotide polymorphism (SNP) with pleiotropic association with leprosy and Crohn's disease (CD) susceptibility alters neutrophil inflammatory responses to NOD2 ligands through altered STAT3 binding and consequent NOD2 expression. Finally, we observe that many neutrophil eQTL reside within regions that have been subject to selection. Collectively, our data advance the understanding of the genetics of gene expression for a pathophysiologically important cell type and role in human disease.

\section{Results}

Defining eQTL in primary human neutrophils. To identify regulatory variants for gene expression in primary human neutrophils, we enrolled 101 healthy adult European volunteers in Oxford, UK (Fig. 1a). Individuals were genotyped on the Human OmniExpress 12v1.0 chip, and we used genome-wide imputation with stringent quality checks to infer additional SNP or simple insertion/deletion genotypes with high confidence. CD16+ neutrophils, isolated by a two-step sequential gradient-density and immunomagnetic sorting procedure yielding a highly purified population, were subjected to whole-transcriptome characterization by hybridization of cRNA to Human HT-12 v4 Expression BeadChips (Illumina). We assessed only genes for which specific probes existed, and which were confidently detected in $>5 \%$ of the cohort (GenomeStudio detection $P<0.01)$. We used a widely adopted linear-additive modelling approach (MatrixeQTL) ${ }^{35}$ adjusting for principal components which has been shown to enhance eQTL discovery and control for technical and demographic heterogeneity $10,19,28,36$.

After genome-wide imputation 3,281 genes (roughly $(\sim) 30 \%$ of 9,147 genes tested and equivalent to 3,675 probes) had one or more identifiable loci within $1 \mathrm{Mb}$ (defined as cis-eQTL) of a probe that was significantly associated with gene expression at a false discovery rate (FDR) threshold of $5 \%$ (Fig. 1b, Supplementary Data 1). The median proportion of variance explained by the most significant variant for each probe, was $16.6 \%$ (interquartile range (IQR) $12.9-26.0 \%$ ) but notably was $>50 \%$ for several genes, such as C4BPA, involved in venous thrombosis $^{37}$, the anti-inflammatory monosodium urate receptor $C L E C 12 A^{38}$ and the antibacterial enzyme lysozyme (LYZ; Additional genes with large effect sizes are highlighted in Fig. 1b). Genes with an eQTL were more highly expressed (median normalized expression 8.14 versus $7.7, P=1.7 \times 10^{-72}$ ) and had greater variance (median variance 0.049 versus 0.029 , $P=1.07 \times 10^{-122}$ ) than genes without an eQTL. The most significant eQTL per gene (denoted as the peak eQTL) clustered around the transcriptional start site (TSS), and the effect sizes increased with proximity to the TSS (Supplementary Fig. 1a) but, annotation and inspection at higher resolution demonstrates distribution of eQTL across gene structures with clustering around TSS and transcription end sites, consistent with previous studies $^{39}$ (Supplementary Fig. 1b).

We did not identify association between any of 168 copynumber variants, inferred from raw genotype calls, and gene expression after correcting for multiple comparisons (Supplementary Data 2).

Comprehensive identification of loci associated with gene expression on non-contiguous chromosomes (trans eQTL) typically requires large sample ${ }^{12}$ sizes due to typically reduced effect sizes of variants acting trans allied to an increased burden of multiple hypothesis testing. Accordingly, we identified a smaller set of 33 genes regulated in trans, at a false discovery rate threshold of $<1 \%$ (Fig. 1b, Supplementary Data 3). These trans eQTL include rs10012416, which we find is a cis-eQTL for CRIPAK (encoding an inhibitor of p21 activated kinase Pak1 important in neutrophil cytoskeletal dynamics involved in phagocytosis ${ }^{40}$ ) and has trans effects on AVP, SPTBN3 and IRF6; and rs10784774, a cis-eQTL for LYZ that is associated with ZNF131 in neutrophils as we previously had reported in monocytes ${ }^{13}$. We note that this study is not powered to identify trans eQTL with weak to moderate-sized effects.

eQTL in genes central to neutrophil biology. We proceeded to examine genes relevant to neutrophil function for eQTL as these may serve as a narrative resource to understanding the role of 
a

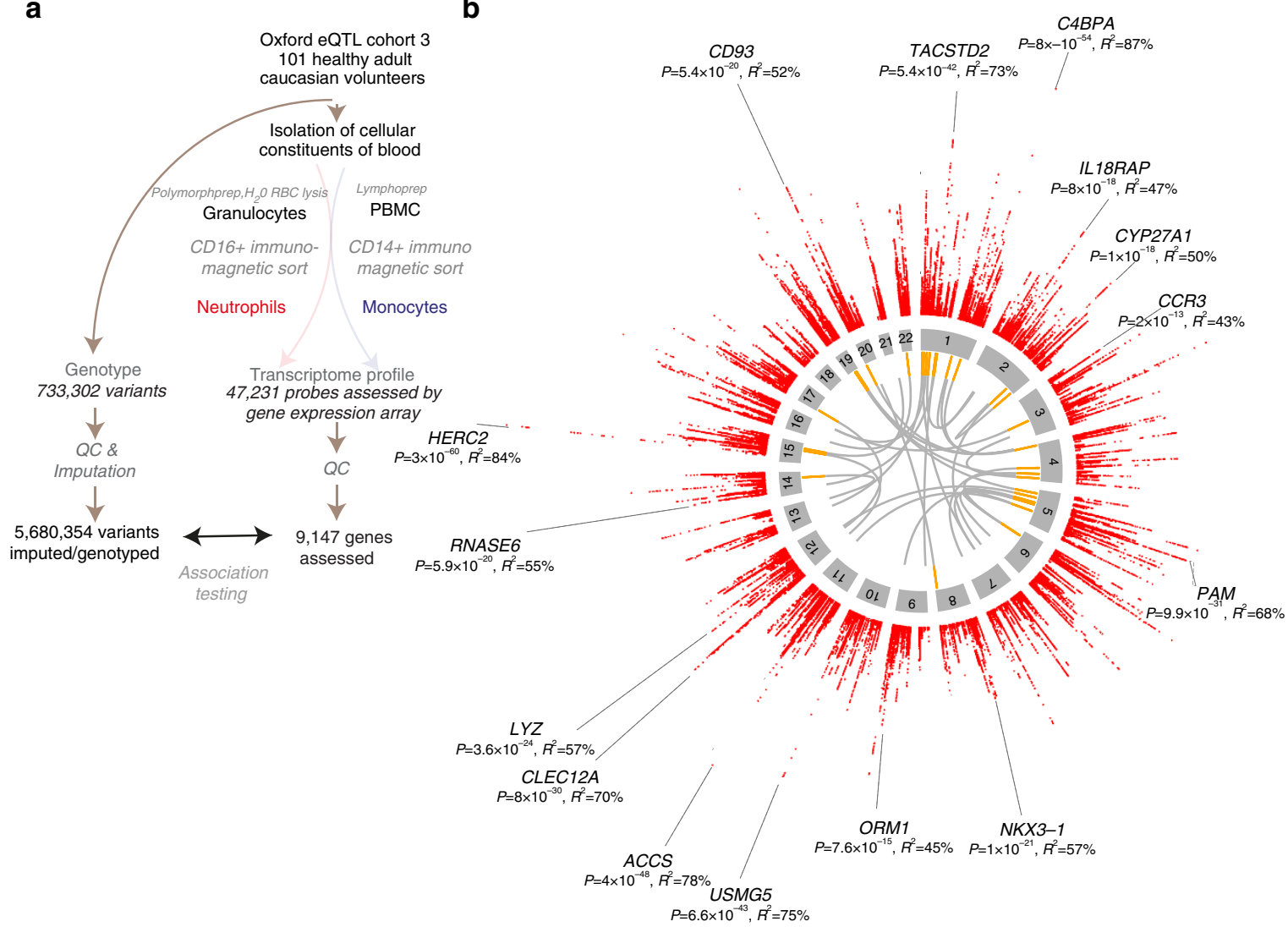

Figure 1 | Study schema and overview of eQTL in primary human neutrophils. (a) Flowchart showing the schema of this study to identify eQTL in human neutrophils from healthy adult volunteers of European ancestry with genotypes determined by array-genotyping and by imputation tested for association with global gene expression in cis (variant $<1 \mathrm{Mb}$ of gene) or trans (variant on non-contiguous chromosome to gene). This analysis was contemporaneous to our previously reported study ${ }^{19}$ of monocytes enabling direct comparison of genetic correlates of gene expression between neutrophils and monocytes. (b) Circos plots for the neutrophil dataset. Outermost rim (red dots) shows a Manhattan plot for significant cis-eQTL (FDR $<0.05)$ with names of genes with large effect sizes (variance explained, $R^{2}>45 \%$ ) or lowest five $P$-values; the second rim (grey boxes) shows the chromosome ideogram with chromosome number within each box; the third rim (orange boxes) indicates a gene to which a significant (FDR $<0.01)$ trans-eQTL was observed; and the innermost spokes (grey) connect trans-acting variants to the gene the variant is associated with.

regulatory variants in neutrophil biology. We assembled a list of 164 genes described, with a verifiable reference, to be involved in a particular aspect of neutrophil function as being involved in aspects of neutrophil function in recent reviews $29,30,41-43$. Because this approach is at risk of narrative bias, we caution against interpretation of these results as evidence of enrichment for genes important in neutrophil function. Of the 113 genes for which an expression probe existed, passed QC and was included in analysis, 104 had an identifiable variant associated with expression $(P<0.05)$ and 47 after false-discovery adjustment $($ FDR $<0.05)$. We observe eQTL for genes involved in most aspects of neutrophil development and function (Fig. 2, Supplementary Data 4). Interestingly, several genes with an eQTL are implicated in Mendelian disorders involving neutrophils. For example rs933222 is associated with expression of $R A C 2$, a gene encoding a Rho GTPase that is part of the NADPH oxidase complex involved in initiation of phagocytosis (Fig. 11) and is involved in neutrophil immunodeficiency syndrome $^{44}$ (OMIM \#608203).

Ingenuity pathway analysis of 975 genes with an eQTL in neutrophils but not in monocytes (Supplementary Fig. 2, and detailed further below), revealed enrichment for functions relating to cell death $\left(P=1.4 \times 10^{-7}\right)$, apoptosis $\left(8.2 \times 10^{-7}\right)$, necrosis $\left(6.4 \times 10^{-6}\right)$; and infection, notably viral infection $\left(4.2 \times 10^{-6}\right)$ and infection of cells $\left(2.2 \times 10^{-5}\right)$. The most significant upstream transcriptional regulator was TP53, which plays a critical role in cell proliferation and apoptosis as well as antimicrobial function highlighting how the consequences of TP53 for the individual may be modulated by eQTL in downstream mediator and effector genes. A number of cytokines were also identified as upstream regulators including IFNB1 $\left(P=5.9 \times 10^{-4}\right), \quad \operatorname{IL} 15 \quad\left(3.0 \times 10^{-3}\right), \quad$ IFNG $\left(4.1 \times 10^{-3}\right)$, CD40LG $\left(6.8 \times 10^{-3}\right)$ and TNF $\left(7.2 \times 10^{-3}\right)$.

Patterns of eQTL in neutrophils and other immune cell types. Regulation of gene expression may be constrained to specific cell types and contexts. To delineate aspects of shared and unique regulatory genomic architecture in neutrophils, we pursued three complementary approaches.

First, we note that $63 \%(2069 / 3281)$ of genes with a cis-eQTL in neutrophils are reported to have a cis-eQTL in the largest blood eQTL meta-analysis to date ${ }^{12}$ (obtained through the bloodeqtl browser) and recent in silico predictions ${ }^{28}$ attribute $20 \%$ (443/ $2188)$ to neutrophils and $90 \%(1969 / 2188)$ as 'generic' (note that a gene may be denoted as neutrophil and generic). Therefore, many eQTL in neutrophils may not have been identified in whole-blood studies, and even when they are, in silico deconvolution of cell types may not establish if a gene has an eQTL in a given cell type. Conversely, $84 \%$ of genes $(415 / 495)$ 
with an eQTL in blood that are bioinformatically ascribed to neutrophils (and tested in our study) have an eQTL in purified neutrophils, providing evidence of cross-study validation. The estimated effect sizes in neutrophils and whole blood for genes with an eQTL in both are only moderately correlated (spearman correlation estimate for 691 genes, rho $=0.54,95 \%$ CI $0.43-0.60$, $\left.P=1.77 \times 10^{-23}\right)$; as we shall present later, directionally opposing eQTL amongst different cells are not uncommon and a

Neutrophil lineage development CEBPA, CEBPB, CEBPG, CSF3R* CEBPD, CEBPE

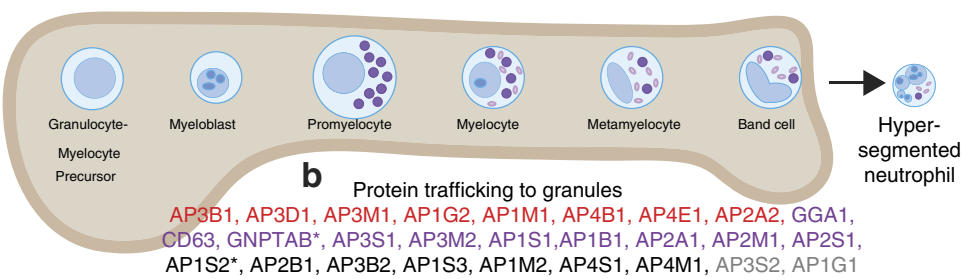

d

Egress from bone marrow and migration towards chemotaxins CXCR4*,

AP3B1, AP3D1, AP3M1, AP1G2, AP1M1, AP4B1, AP4E1, AP2A2, GGA1, AP1S2*, AP2B1, AP3B2, AP1S3, AP1M2, AP4S1, AP4M1, AP3S2, AP1G1

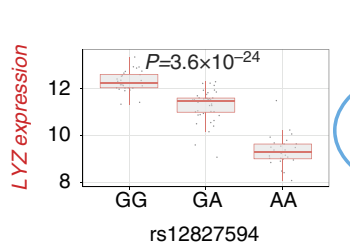

C
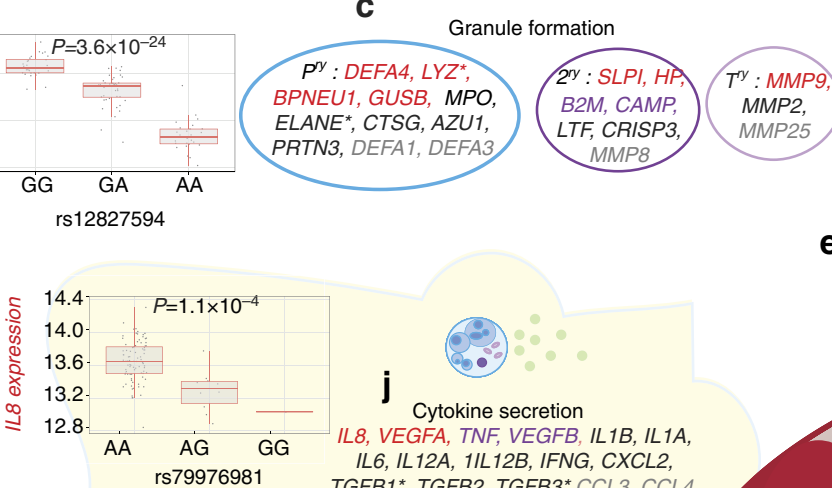

Cytokine secretion IL8, VEGFA, TNF, VEGFB, IL1B, IL1A ILG, IL12A, 1IL12B, IFNG, CXCL2 TGFB1* TGFB2, TGFB3*

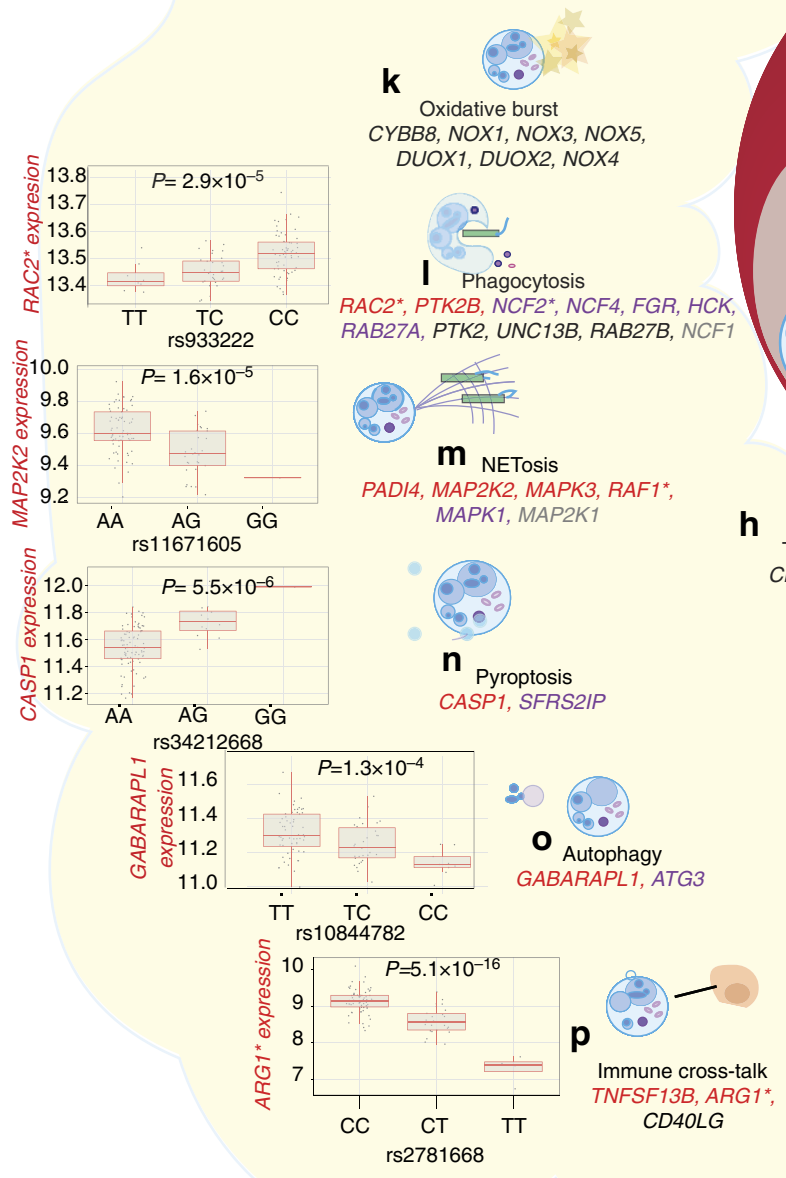

Key
eQTL observed( FDR $<0.05)$ QTL observed( $F D R<0$. No 0 TTL 0 ( No eQTL observed

$r^{*}$ gene involved in Mendelian disorder

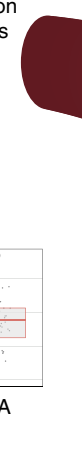

e Capture and rolling

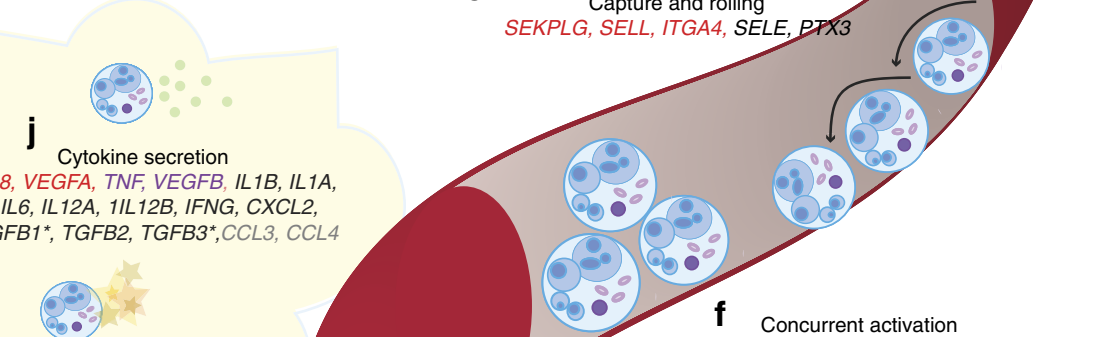
SRC*, PIK3CB, MAPK14, SYK, PIK3CA, PIK3R5,
PIK3CG, MAPK 13, PIK3C2B, PIK3C2G, MAPK11,

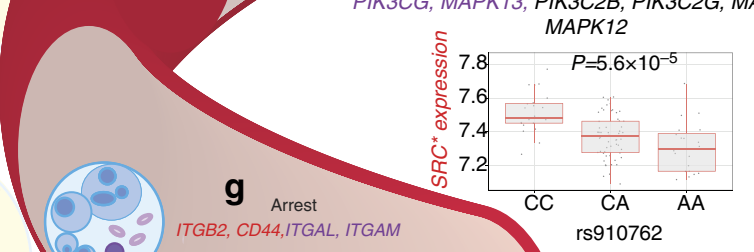

Transmigration CD99, LOC

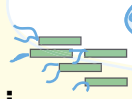

I Pattern recognition and migration along ligand gradient

FPR1, FPR2, CARDQ*, TLR4, CLEC7A, NLRP3, CLEC12A, FCGR3A, FCGR3B, IFNGR1, IFNGR2, * 7.8 CLEC12A, FCGR3A, FCGR3B, IFNGR1, IFNGR2, * 7.8
LTB4R, CRAR1, TLR1, TLR2, TLR5, TLR9, MYD88, 7.6 IRAK4, NLRC4, IRAK1, LTB4R2, IKBKG
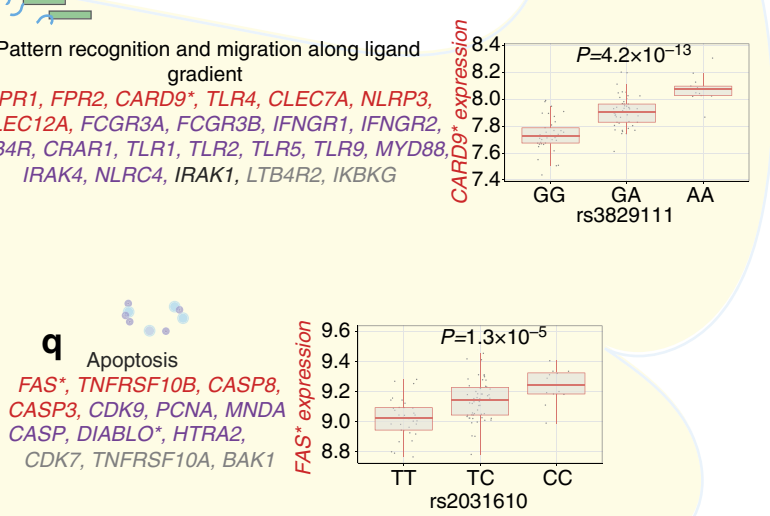
this may plausibly affect in silico effect size estimates. Second, for $40 \%(261 / 646)$ of genes with a cis-eQTL in mouse CD4 + T cells and/or neutrophils ${ }^{25}$ whose human homologues we tested in humans, we observe a significant cis-eQTL in neutrophils (a 5.72fold enrichment over random expectation (646/9147), $\left.P=1.02 \times 10^{-80}\right)$. Third, we compared genes with an eQTL in neutrophils to those that have been reported previously in other primary immune cells: CD4 T-cells, regulatory $\mathrm{T}$ cells ${ }^{17}$ and $\mathrm{B}$ cells $^{13}$ (grouped as lymphoid cells) or monocytes and monocyte-derived dendritic cells ${ }^{14-16,19}$ (grouped as myeloid cells). Of the 3281 genes with an eQTL in neutrophils, 1,671 (51\%) had an eQTL in both myeloid and lymphoid cells, 823 $(25 \%)$ in non-neutrophil myeloid cells, $337(10 \%)$ in lymphoid cells and 450 have no reported eQTL in either myeloid or lymphoid cells (Supplementary Fig. 3). Although our analysis neither assesses whether the same genetic variant regulates gene expression in all cell types nor whether the effect is the same, it demonstrates that, for at least $14 \%$ of the genes in which we observe an eQTL, we have identified novel regulatory variants and shown the utility of eQTL mapping in primary neutrophils.

To further examine the effect of cell type on eQTL, we performed a detailed analysis of monocytes and neutrophils isolated contemporaneously from 99 caucasian donors in which we confined analysis to 8,362 genes expressed in both cell types. We identified 400,783 unique variant-gene associations across both cell types: 87,276 variants for 1031 genes in both neutrophils and monocytes, 118,817 variants for 2,847 genes in neutrophils and 194,690 variants for 3,674 genes in monocytes (Fig. 3a). Higher expression of a gene in neutrophils compared with monocytes was a positive predictor of whether an eQTL was present in neutrophils or not, but normalized probe intensity explains just $\sim 2 \%$ of variance and there are many genes in which an eQTL is observed in only one of neutrophils or monocytes despite similar levels of gene expression. The effect size of regulatory variants in both monocytes and neutrophils was larger for eQTL seen in both cell types compared with those seen only in one cell type $\left(P=7.4 \times 10^{-196}\right.$, Supplementary Fig. 4) confirming previous observations ${ }^{13}$. Interestingly, whereas the number of unique eQTL is greater in monocytes, eQTL effect sizes were larger in neutrophils than in monocytes regardless of whether the eQTL was seen in both cell types $\left(P=1.9 \times 10^{-19}\right)$ or just one $\left(P=9 \times 10^{-200}\right)$. This likely reflects a proportionally greater impact of genetic variation in governing gene expression in neutrophils that recapitulates their shorter lifespan and reduced exposure to environmental modifiers.
We highlight three patterns of cell environment-modifying genetic effects on gene expression in addition to apparent celltype constraint of regulatory activity. First, although 1,939 genes have at least one eQTL in both cell types (and 1,031 share an eQTL), for 908 genes the peak regulatory variants are different and for $840(93 \%)$ of these, independent $\left(r^{2}<0.2\right)$ demonstrating that the same gene may have independent regulatory variants of varying strengths and directions in different cell types. An example for the OSCAR gene is shown in Fig. 3b. Conversely a particular variant may show pleiotropy in the gene it regulates in neutrophils and monocytes. About 4.5\% (12,661/278,200) of variants that regulate a gene in one cell-type are associated to a different gene in the other cell type. For example, rs35244261 is associated with levels of ATM in neutrophils and NPAT in monocytes (Fig. $3 c$ ). In gene-dense regions this could be due to cell-type conditioning the effect of one or more variants that have regulatory effects on nearby genes. The third pattern we highlight is pleiotropy in direction of effect of a variant in different cell types on the same gene. We identified 2,823 variants with significant but directionally opposing effects on 66 genes in both cell types (Fig. 3d). Several are notable for the variant being associated with a disease directly or through a linked variant such as rs8066560 (TOM1L2, Parkinson's disease), chr18:3375159:D (Fig. 3e, ELP2, oesophageal squamous cell carcinoma), rs74058715 (PADI4, rheumatoid arthritis), and rs1981760 (NOD2, leprosy and CD), the latter two described in detail below. Collectively, the comparison of monocyte and neutrophil eQTL supports a model of widespread interaction between cellular milieu and genetic factors in regulation of gene expression even amongst cells of similar (myeloid) lineage. Moreover, delineation of cell-type restriction of eQTL in neutrophils may provide a tool to understand their involvement in variantphenotype association.

Epigenetic mechanisms of eQTL in neutrophils and monocytes. The local genomic environment of a regulatory variant is cell-type dependent and this may modify variant effect. Moreover, as has been shown for complex traits, leveraging epigenetic mark information may help fine-map genotype-phenotype associations ${ }^{45}$. Therefore, to elucidate the role DNA methylation and histone modification play in genetic effects on gene expression, we compared eQTL maps in neutrophils and monocytes to maps of DNA methylation and several histone modifications generated by whole-genome bisulfite sequencing or ChIP and sequencing (ChIP-Seq), respectively, in neutrophils and monocytes from 4 to

\footnotetext{
Figure 2 | eQTL in genes involved in neutrophil biology. As a narrative resource, we compiled a list of 164 genes of importance in neutrophil biology 29,30,41-43 and illustrate their role together with eQTL information (denoted be gene colour). Probes for 113 genes passed QC: 9 genes had no eQTL (black gene names) and 104 had an eQTL ( 47 with FDR $<0.05$ red gene names, 57 with $P<0.05$ but FDR $>0.05$ purple gene names). Genes involved in nearly every aspect of neutrophil biology have identifiable eQTL and several are involved in Mendelian disorders (asterisked genes) for example RAC2 (neutrophil immunodeficiency syndrome) and FAS (autoimmune lymphoproliferative syndrome type 1A). (a) Granulocyte-monocyte progenitor cells (GMP) respond to GM-CSF stimulation of a receptor encoded by CSFR3 to form neutrophils under the instruction of CEBP $\alpha$ and other CEBP transcription factors. Neutrophil development through various stages is coincidental with expression of proteins that traffic to primary, secondary and tertiary granules. (b) AP heterotetramers are involved in trafficking of proteins such as LYZ (involved in renal amyloidosis) and MMP9 (involved in metaphyseal osteolysis, nodulosis and arthropathy) to (c) primary, secondary and tertiary granules. (d) Hypersegmented neutrophils egress from the bone marrow in response to IL-8 and SDF-1, and are (e) captured on endothelium within blood vessels through interaction of selectins and ligands such as SEKPLG and SELL. (f) Capture, rolling and eventual arrest on endothelial surfaces is associated with activation, mediated in part by PI-3 and MAPK pathways. (g) Arrest of neutrophils is mediated by $\alpha 1 \beta 2 / \alpha \mathrm{M} \beta 2$ integrin heterodimers encoded by ITGAL, ITGBA2, ITGAM and (h) rapid para- and trans-cellular migration ensues. (i) Neutrophils express pattern recognition receptors and receptors for host derived molecules allowing detection of bacteria and effective migration. In tissues, neutrophil response includes (j) cytokine secretion, (k) oxidative burst, (I) phagocytosis, (m) production of neutrophil extracellular traps (NETosis), (n) pyroptosis, (o) autophagy, (p) crosstalk with other immune, and ( $\mathbf{q})$ eventual cell death via apoptosis should cell death not have eventuated from another response. Box lower and upper border denote 25 th and 75th centiles, respectively, central line denotes median and whiskers extend to $1.5 \times 1 \mathrm{IQR}$. In all cases 101 donor replicates shown.
} 
a

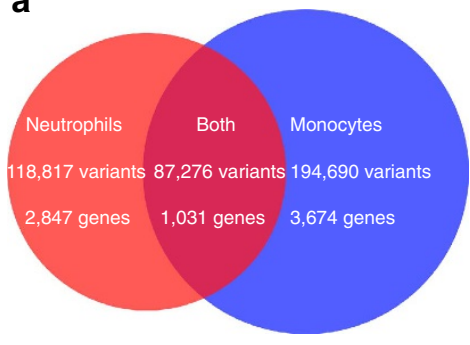

d

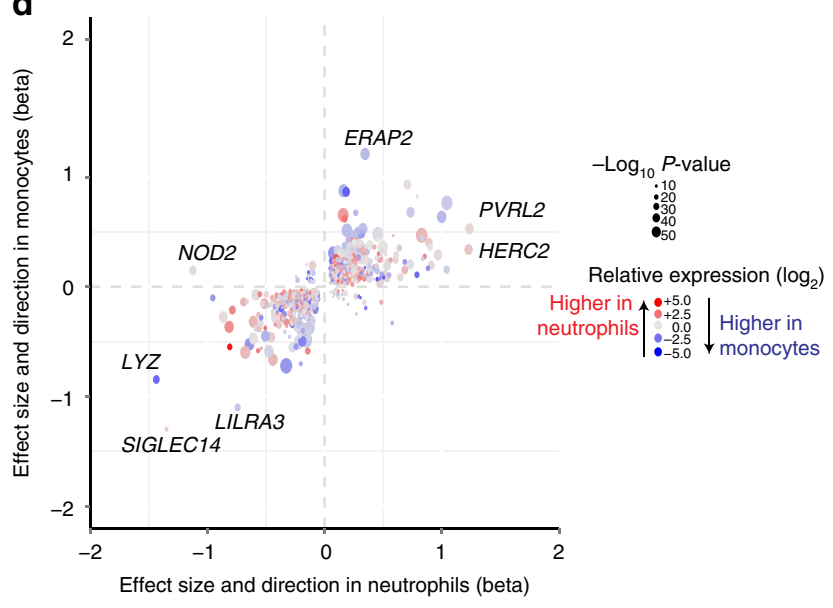

b

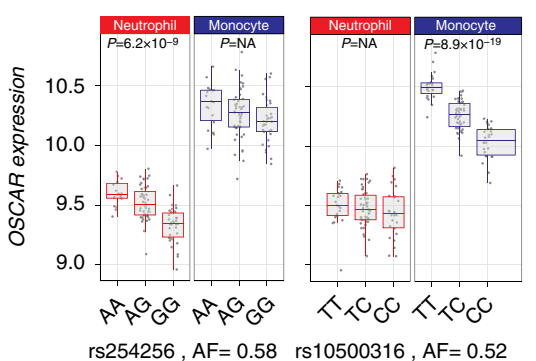

C

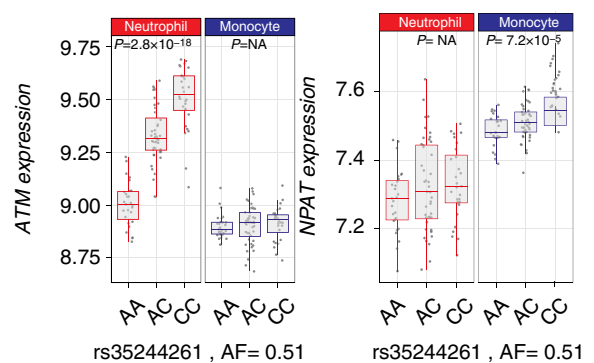

e

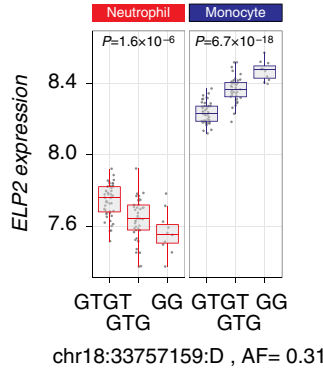

Figure 3 | Shared and cell-type-specific cis-eQTL in neutrophils and monocytes. (a) Venn diagrams showing the number of variants and genes with cis-sQTL by cell type. Examples of cell-type constraint include (b) independent variants associated with expression of the same gene, OSCAR, specific to either neutrophils or monocytes (lead eSNPs rs254256 and rs1050031, respectively, $r^{2}<0.02$ ). Conversely, (c) a single variant may be associated with different genes in each cell type as shown for rs35244261 (associated with elevated expression of ATM in neutrophils, and NPAT in monocytes). (d) Amongst 1,939 genes that have an eQTL in both cell types, 1,031 involve the same variant and are plotted with the effect size in neutrophils and monocytes (shown on $x$ - and $y$ axes, respectively). Colour denotes the relative expression in neutrophils and monocytes, and size denotes the minimum $P$-value of an eQTL for that gene. Several eQTL show divergent direction of effects on the gene they regulate including NOD2, THBD, TSTD1, TOM1L2 and ELP2 (latter shown in e). Genes with large effect sizes are highlighted, including $H E R C 2$, the gene responsible for iris colour, which has amongst the most significant eQTL of all genes in neutrophils. $P$-values $>0.05$ are denoted as $P=$ NA. Box lower and upper border denote 25th and 75th centiles, respectively, central line denotes median and whiskers extend to $1.5 \times I Q R$. In all cases 101 donor replicates shown.

8 individuals by the BLUEPRINT consortium ${ }^{46}$. As may be predicted by their shared ontology, monocytes and neutrophils have substantial overlap in regions of the genome that are methylated or subject to histone modifications, although this differs by the specific combination of epigenetic mark (Supplementary Data 5). Relative to all imputed variants within $1 \mathrm{MB}$ of a gene $(4,812,340$ variants near a neutrophil-expressed gene tested for being a cis-eQTL), we observed marked and significant enrichment of peak cis-eQTL in hypomethylated regions $\left(P_{\text {neut }}=2.14 \times 10^{-107}, \quad P_{\text {mono }}=2.52 \times 10^{-124}\right)$ and depletion in hypermethylated regions $\left(P_{\text {neut }}=6.36 \times 10^{-46}\right.$, $P_{\text {mono }}=7.55 \times 10^{-23}$ ) in the concordant cell type that is, neutrophil eQTL in neutrophil hypomethylated regions (Fig. 4a,c, Supplementary Data 6). Similarly, as shown in Fig. 4b (and Supplementary Data 6), peak cis-eQTL were enriched in regions with histone marks associated with promoter activity (H3K4me3; $\quad P_{\text {neut }}=8.23 \times 10^{-231}$, $P_{\text {mono }}=1.31 \times 10^{-165}$ ), active or poised enhancers $(\mathrm{H} 3 \mathrm{~K} 27 \mathrm{Ac}$; $P_{\text {neut }}=4.85 \times 10^{-230}, \quad P_{\text {mono }}=1.34 \times 10^{-80}, \quad$ H3K $4 \mathrm{mel}$; $\left.P_{\text {neut }}=2.23 \times 10^{-308}, \quad P_{\text {mono }}=4.84 \times 10^{-229}\right) \quad$ or activation $\left(\mathrm{H} 3 \mathrm{~K} 36 \mathrm{me} 3 ; P_{\text {neut }}=2.01 \times 10^{-238}, P_{\text {mono }}=3.22 \times 10^{-196}\right)$ and depleted in regions associated with repressive histone modifications $\quad\left(\mathrm{H} 3 \mathrm{~K} 27 \mathrm{me} 3 ; \quad P_{\text {neut }}=3.78 \times 10^{-3}\right.$, $P_{\text {mono }}=2.59 \times 10^{-2}$ and H3K9me3; $\quad P_{\text {neut }}=3.35 \times 10^{-4}$,
$P_{\text {mono }}=2.57 \times 10^{-2}$ ). These data are replicated by an orthogonal approach, in which, despite vastly fewer regions, we observe 14-fold enrichment of neutrophil eQTL in neutrophil enhancer regions $\left(P=3.03 \times 10^{-15}\right)$ and 6-fold enrichment of monocyte eQTL in monocyte enhancer regions $\left(P=3.58 \times 10^{-17}\right)$ using enhancer maps generated by CAGESeq in FANTOM $5^{47,48}$. These data support a model of epigenetic environment-modifying regulatory effects in different cell types. An example for the PADI2 gene is shown in Fig. 4d,e.

Mechanisms by which regulatory variants operate include alteration in transcription factor binding. Neutrophil and monocyte peak eQTL are 3.1- and 2.87-fold enriched $\left(2.70 \times 10^{-118}\right.$ and $1.92 \times 10^{-123}$, Supplementary Data 6), respectively, in regions bound by a transcription factor in cell lines studied in the ENCODE project ${ }^{49}$. Expression levels of $44 \%$ $(415 / 943)$ of reported human transcription factors ${ }^{50}$ that are expressed in both cell types differ by $>0.5 \log _{2}$ between neutrophils and monocytes (Supplementary Data 7), making it plausible that alteration in transcription factor binding sites in addition to differences in transcription factor abundance could lead to eQTL being observed in one cell type and not the other. About 1\% (52 in neutrophils, 35 in monocytes) of peak eQTL lie within microRNA binding sites, a 3.44- $\left(P=1.05 \times 10^{-9}\right)$ and 4.14-fold $\left(1.01 \times 10^{-16}\right)$ enrichment for neutrophils and 
a

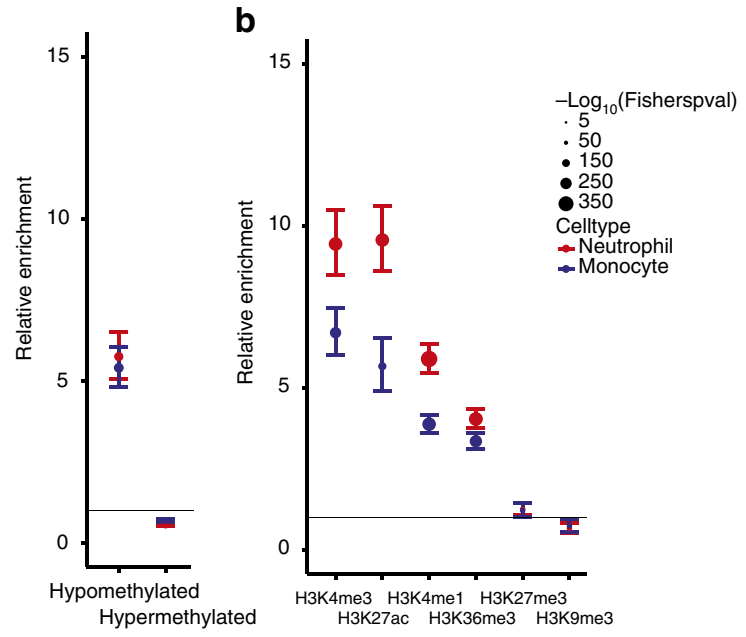

d

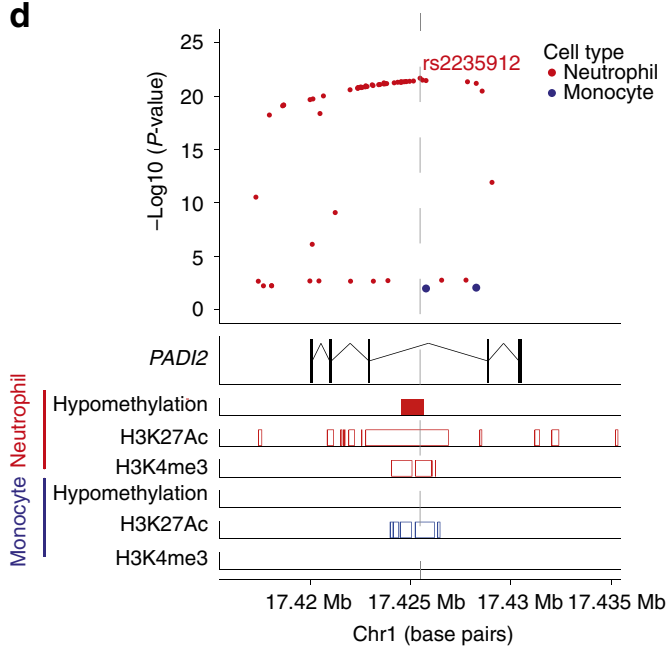

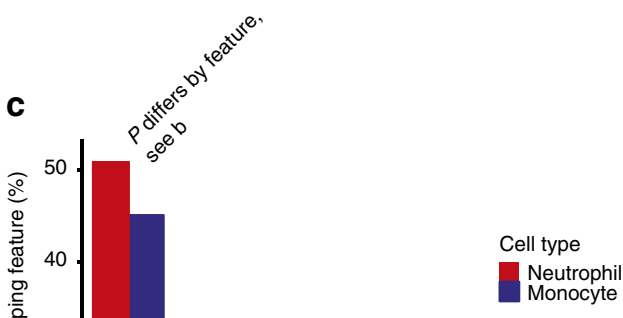

Neutrophi

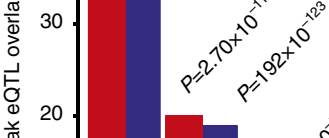

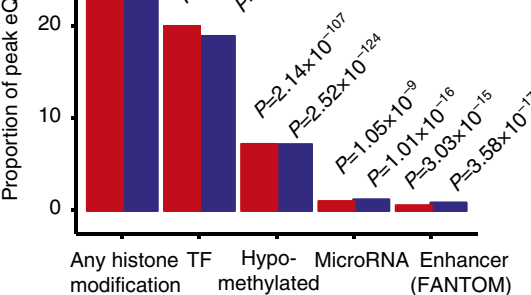

e

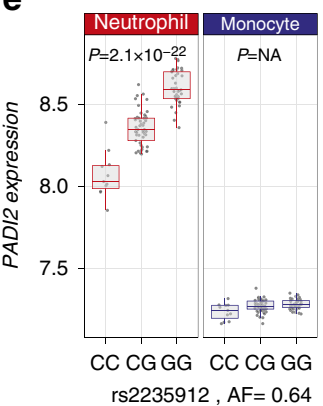

f

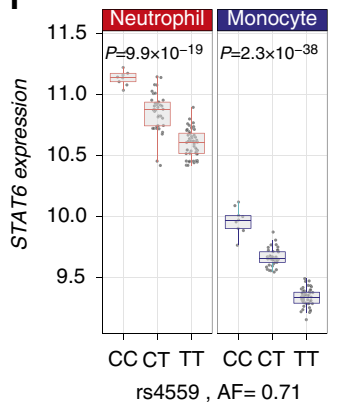

Figure 4 | Epigenetic basis of eQTL in neutrophils and monocytes. We integrated eQTL data with whole-genome bisulfite sequencing and chromatinimmunoprecipitation sequencing data from primary neutrophils and monocytes from 4 to 8 individuals from the BLUEPRINT Consortium. (a) Enrichment of cis-eQTL in neutrophils and monocytes, in hypo- and hypermethylated regions of the genome in concordant cell types relative to all SNP's tested for eQTL. (b) Enrichment of neutrophil and monocyte cis-eQTL relative to all variants tested for colocalisation in regions of the genome associated with modified histones based on ChIP-Seq of modified histones in neutrophils and monocytes. (c) Proportion of cis-eQTL in neutrophils $(n=3,774)$ and monocytes $(n=4,668)$ that overlie modified histones in the concordant cell type (BLUEPRINT), transcription factor binding sites in cell lines (ENCODE), sites that are hypomethylated in the concordant cell type (BLUEPRINT), target sites of conserved miRNAs with high mirSVR scores (microRNA.org) or CAGE-seq defined enhancer sites in neutrophils and monocytes (FANTOM5). Note that an eQTL may overlap more than one feature. (d,e) An example of a cell-typespecific eQTL possibly attributable to methylation differences in cell types is rs2235912, a site which is in an intron of PADI2 that is hypomethylated in neutrophils but not monocytes. This locus is marked by the activating histone marks H3K27Ac and H3K4me3 in neutrophils but in monocytes has a smaller region marked by H3K27Ac only. As shown in $\mathbf{e}$, the gene is more highly expressed in neutrophils than monocytes, perhaps due to the greater activating histone marks in neutrophils. The G allele creates an additional putative CpG site, which may explain the allele being associated with higher PADI2 expression relative to the $\mathrm{C}$ allele. (f) STAT6 is an example of a gene with an eQTL in both monocytes and neutrophils where the eQTL lies in a predicted binding region for mir-18b, a microRNA. Tails in $\mathbf{a}, \mathbf{b}$ show $95 \% \mathrm{Cl}$ of the enrichment estimate. In e,f, box lower and upper border denote 25 th and 75 th centiles, respectively, central line denotes median and whiskers extend to $1.5 \times \mathrm{IQR}$. In all cases 101 donor replicates shown. $P$-values $>0.05$ are denoted as $P=N A$.

monocyte respectively (Supplementary Data 6 and 8). For example, rs4559 is a common variant associated with STAT6 expression (Fig. 4f) in both cell types and is within the binding region of miR-18b. We note that although this analysis does not take into account whether the microRNA is expressed in the cell-type, the results are consistent with some eQTL having their effect through microRNA-directed transcriptional gene silencing 51 .
Neutrophil eQTL are enriched for disease-associated variants. eQTL mapping can provide functional insight into the basis for genetic association of complex traits. We systematically examined eQTL in neutrophils for association with complex traits. Of all eQTL in neutrophils $7.5 \%(14,390 / 191,767)$ are associated with one or more of 327 traits (of 1,138 unique disease/traits curated in the NHGRI GWAS catalogue ${ }^{52}$ ) directly or through linkage $\left(r^{2}>0.8\right)$ with the trait-associated variant (Supplementary 
Data 9). This represents a 2.7 -fold (95\% CI 2.67-2.77, $P<2.2 \times 10^{-16}$ ) enrichment relative to all SNPs originally tested for association with gene expression (eQTL). Notable examples are highlighted in Fig. 5a. Reciprocally, 7.8\% (14,390/
$183,124)$ of trait-associated variants are, or tag, eQTL in neutrophils.

Collation of GWAS traits into disease categories demonstrates that enrichment is observed for gastrointestinal disorders
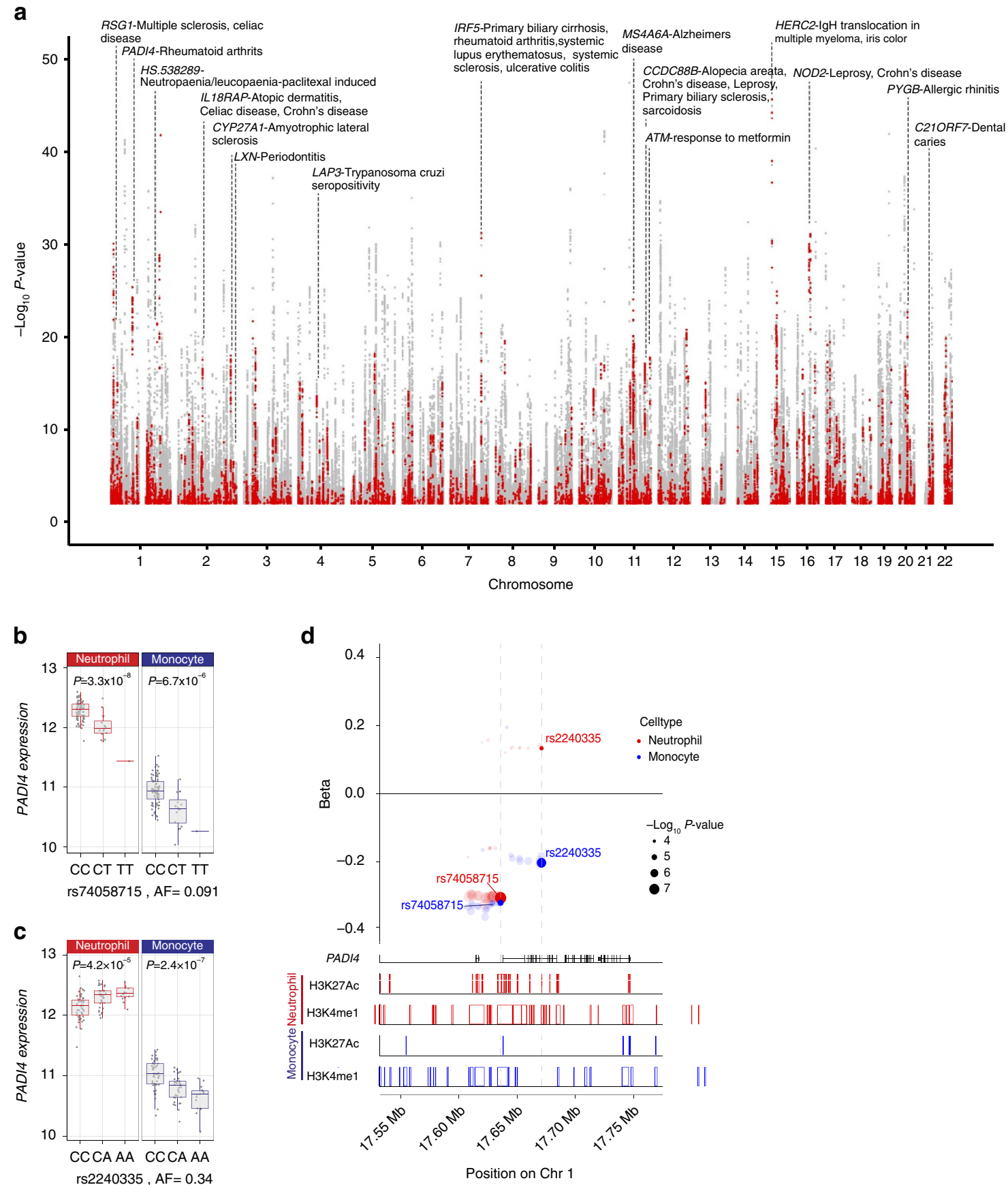

Figure 5 | eQTL in neutrophils and their association with complex disease or trait. (a) Manhattan plot showing eQTL in neutrophils highlighting those associated with or in linkage disequilibrium $\left(r^{2}>0.8\right)$ with a disease/trait associated variant listed in the NHGRI GWAS catalog. Each point denotes a single $\mathrm{eQTL}$ and is coloured red if the locus is associated with a complex trait or grey if not. (b-d) An example in PADI4 demonstrates how integrated analysis of an eQTL informs understanding of rheumatoid arthritis (RA) risk. Expression level of PADI4 is associated with two independent variants, (b) rs 2240335 and (c) rs74058715 $\left(r^{2}=0.02\right)$. rs2240335-A, the derived allele, is associated with elevated expression of PADI4 in neutrophils and diminished levels in monocytes. rs7405871-T is associated with reduced PADI4 expression in neutrophils and monocytes. rs2240335 is in near-complete linkage with rs2301888, and this locus is associated with RA risk ${ }^{54}$ (d) Compiled plot showing effect size estimate (beta) for variants associated with PADI4 expression in neutrophils and monocytes (upper panel), relative to genic structures (track two), and BLUEPRINT ChIP-Seq reads for two histone marks in neutrophils (tracks three and four) and monocytes (tracks 5 and 6) for H3K27Ac and H3K4me1 demonstrating that rs2240335 lies in a region marked by both H3K27Ac and H3K4me1, a marker of active enhancers, in neutrophils but not in monocytes. In b,d, box lower and upper border denote 25th and 75th centiles, respectively, central line denotes median and whiskers extend to $1.5 \times \mathrm{IQR}$. In all cases 101 donor replicates shown. 
(7.2-fold enrichment; $P<2 \times 10^{-308}$ ), allergy (6.3-fold enrichment; $P=1.3 \times 10^{-117}$ ), autoimmune (6.3-fold enrichment, $P<2 \times 10^{-308}$ ) and infectious diseases (3.1-fold enrichment, $P=7.9 \times 10^{-137}$ ) grouped together or disaggregated for viral (3.7-fold enrichment, $\left.P=1.8 \times 10^{-56}\right)$, parasitic (5.1-fold enrichment, $\left.P=6.4 \times 10^{-96}\right)$ and bacterial (3.1-fold enrichment, $P=2 \times 10^{-34}$ ) diseases (Supplementary Data 10 ).

We note that many of the eQTL observed in neutrophils may also be observed in monocytes (Supplementary Fig. 5) reinforcing the need for additional follow-up to resolve complexity of how a variant may predispose to disease through effects in one or more cell types. We therefore explored two eQTL in greater detail to demonstrate how integrated analysis can provide novel insights into disease.

An eQTL of PADI4 affects rheumatoid arthritis susceptibility. Rheumatoid arthritis is a systemic autoimmune disease afflicting $\sim 0.5-1 \%$ of adults in which autoimmune destruction of synovial joints occurs ${ }^{53}$. A major feature of disease is the presence of autoantibodies directed against citrullinated proteins. We found that 13 of 101 loci (a threefold enrichment compared with background, 95\% CI $1.5-5.4, P=8 \times 10^{-4}$ ) recently reported to be associated with rheumatoid arthritis risk in the largest metaanalysis to date $\mathrm{e}^{54}$ are eQTL in neutrophils. These include rs2240335, an eQTL for PADI4 that is in near-complete linkage disequilibrium with $\mathrm{rs} 230188\left(r^{2}=0.93\right.$ in our data set). The A allele at rs2240335 is associated with elevated risk of rheumatoid arthritis and together with rs230188 is a genome-wide significant correlate of rheumatoid arthritis risk ${ }^{54}$. Intriguingly, rs2240335-A is associated with increased expression of PADI4 in neutrophils but reduced expression in monocytes (Fig. 5c). rs74058715-T, a nearby SNP independent to rs2240335 $\left(r^{2}=0.02\right)$, is associated with reduced PADI4 expression in both cell types providing an additional instrument to probe the role of PADI4 in neutrophils in rheumatoid arthritis risk (Fig. 5b). Examination of histone modifications in the region show that rs 2240335 lies in a region marked by histone 3 lysine 27 acetylation (H3K27Ac) and histone 3 lysine 4 monomethylation ( $\mathrm{H} 3 \mathrm{~K} 4 \mathrm{me} 1)$ in neutrophils but not in monocytes whereas rs74058715 is in a region marked in both cell types (Fig. 5d), consistent with the cell type in which the eQTL is seen and suggesting rs2240335 as the functional variant as opposed to rs230188. Conditioning on rs2240335 does not reveal a secondary eQTL peak. Following from this prediction, rs74058715-T is nominally associated with reduced rheumatoid arthritis risk $(P=0.05$ in the largest meta-analysis of rheumatoid arthritis) showing directional consistency. We note that rs74058715 tags at least six other SNPs (linkage disequilibrium (LD) $>0.8$ ) that are nominally associated with rheumatoid arthritis, and therefore may not itself be the causal SNP. Subsequent to oxidative responses by stimuli including rheumatoid factor, PADI4 post-translationally citrullinates histones and other proteins initiating NETosis, a process shown to be central to rheumatoid arthritis pathogenesis ${ }^{55-57}$. Because PADI4 expression is confined to neutrophils and monocytes ${ }^{58}$, these data support a model in which rs230188 tags rs2240335-A, which alters PADI4 expression and rheumatoid arthritis risk likely through its actions in neutrophils.

Functional basis of rs1981760 in leprosy and Crohn's disease. Leprosy, a disease caused by the infectious agent Mycobacterium leprae, and $\mathrm{CD}$, an autoimmune inflammatory bowel disease, show profound overlap in genetic architecture ${ }^{59}$. The ancestral T allele of rs1981760 is associated with increased susceptibility of leprosy, particularly multibacillary disease ${ }^{60}$ and yet has weak protective effects on $\mathrm{CD}$, independent to the major $\mathrm{CD}$-associated missense mutations ${ }^{59}$. We observed a strong association between rs1981760-T and reduced expression of both NOD2 $\left(P=8 \times 10^{-30}\right.$, variance explained $\left.=39 \%\right)$, and the adjacent gene SNX20 $\left(P=7.8 \times 10^{-10}\right)$ in neutrophils and conversely elevated expression in monocytes $\left(P=3.3 \times 10^{-10}\right)$ (Fig. 6a,b). The frequency of rs1981760-C is strikingly differentiated in Asians compared with other populations (78\% in Asians versus $26 \%$ in Europeans from the $1000 \mathrm{G}$ project; Fig. $6 \mathrm{c}$ ). To further test the mechanism of this finding and functional consequences, we enrolled an independent cohort of 23 individuals. We were able to replicate the observed eQTL by quantitative PCR with two independent probe sets (Fig. 6d). The rs1981760 polymorphism is reported to alter STAT3 binding in some ENCODE cell lines. We note that rs1981760 is in complete LD with rs9302752, the SNP first reported to be associated with leprosy and $\mathrm{CD}$, but since rs1981760 is the primary eQTL signal, and in view of it potentially modifying transcription factor binding, we pursued rs1981760. Conditioning on rs1981760 does not reveal a secondary eQTL peak. To test whether the polymorphism alters STAT3 binding in neutrophils we performed ChIP with an antibody directed against STAT3 in four heterozygotes. Using allele-specific probes in a digital droplet PCR reaction designed to accurately quantify the number of each allele, we observed significantly fewer copies of immunoprecipitated DNA containing $\mathrm{T}$ alleles than $\mathrm{C}$ alleles after ChIP in each individual (Fig. 6e, Supplementary Data 11) consistent with the hypothesis that the $\mathrm{T}$ allele reduces STAT3 binding either alone or potentially in a complex with other transcription factors. Moreover, STAT3 is markedly differentially expressed in neutrophils and monocytes (Fig. 6f). Finally, we stimulated neutrophils from individuals with the NOD2 ligand muramyl dipeptide (supplemented with Pam3-CSK4 as a synergistic agonist). Individuals with the $\mathrm{CC}$ genotype expressed significantly greater levels of mRNA for IFNB $(P=0.02$; Fig. $6 \mathrm{~g})$ after stimulation. These data demonstrate the rs1981760 affects NOD2 expression and subsequent interferon $\beta$ (IFNB) responses to its ligand. Notably, eQTL in neutrophils are enriched for genes in the IFNB network $\left(P=5.9 \times 10^{-4}\right)$. Therefore these data suggest that type- 1 interferons and neutrophils may be involved in leprosy as has been shown in tuberculosis ${ }^{61}$.

Neutrophil eQTL are enriched in regions of natural selection. Neutrophils are an evolutionarily ancient cell. In light of the rs1981760 observation, we systematically examined whether regulatory variants in neutrophils overlap regions that are identified as being subject to selection in systematic human surveys $^{62}$. Indeed, 1,527 variants that are eQTL for a total of 39 genes lie in regions that have been subject to selection in humans (Supplementary Data 12), a 2.4-fold (95\% CI 2.33-2.6) enrichment $\left(P=1.04 \times 10^{-195}\right)$ relative to the 20,464 variants tested in our study that lie in annotated regions of selection. Many of the genes showing eQTL driven by variants in such regions are known to be involved in leukocyte biology; for example, the chemokine receptors CCR1 (ref. 63) and CCR3, or GUSB, a gene in which mutations cause mucopolysaccharidosis VII (ref. 64) in which recurrent respiratory infections are common. Others, such as HERC2 involved in eye colour $^{65}$ and for which the second strongest eQTL in neutrophils is observed, may suggest new hypotheses for operative selective forces and the cells to yield the patterns observed. Therefore, regulatory variants in neutrophils are likely to have been subject to selection, reinforcing their biological relevance.

In conclusion, these data illustrate the utility of eQTL mapping in different leukocyte subsets and integration with epigenetic and disease-association data to reveal mechanisms of disease. 
a
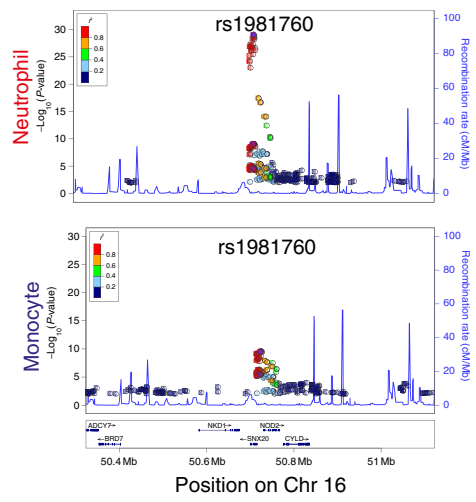

C

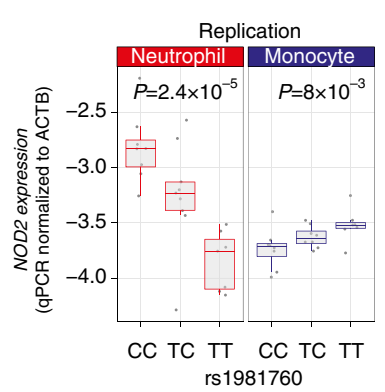

g

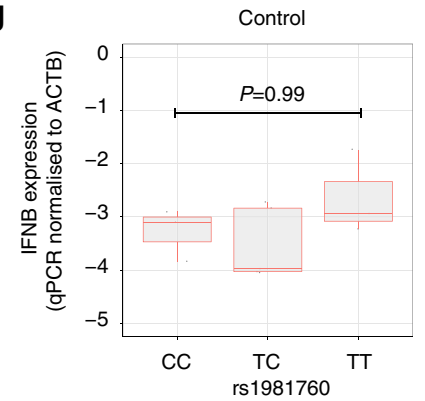

b

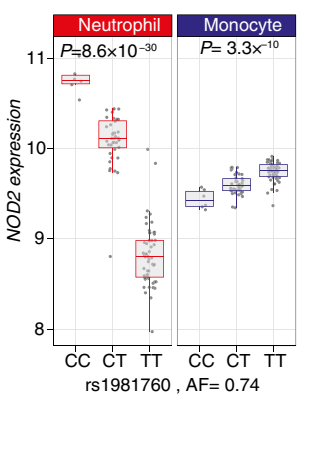

e
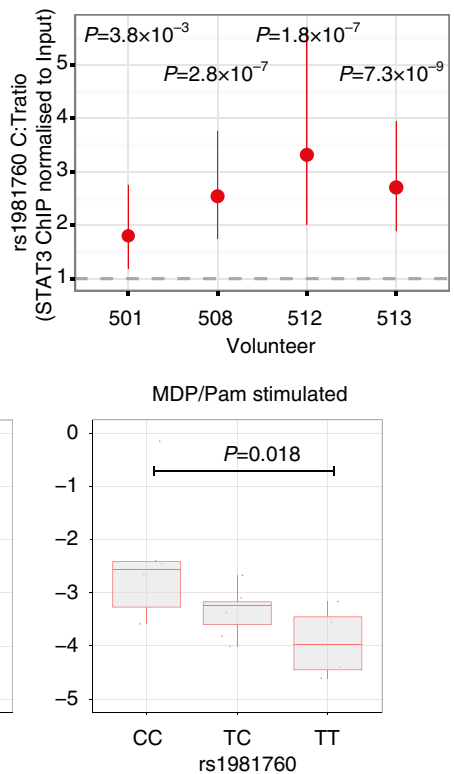

c

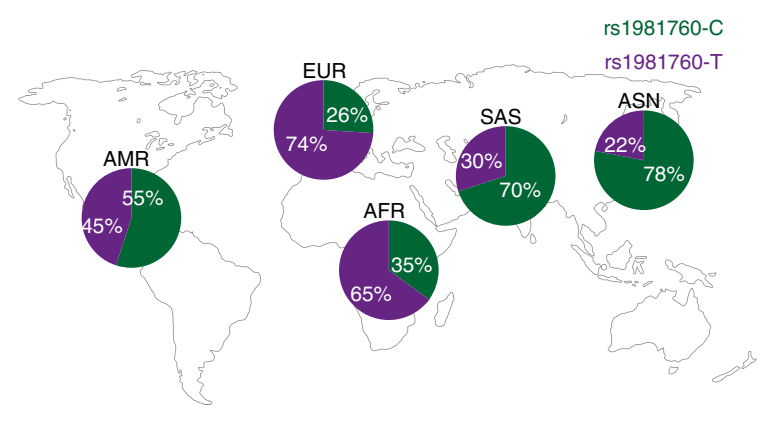

f

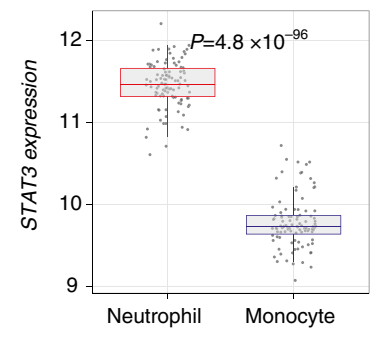

Figure 6 | eQTL in NOD2 and their involvement in Leprosy and Crohn's Disease(CD). (a) Regional association plots for rs1981760 and NOD2 in Neutrophils (top) and monocytes (bottom). (b) The C (derived) allele at rs1981760, a variant located in an intergenic region between NOD2 and SNX20, is associated with elevated NOD2 expression in neutrophils and reduced NOD2 expression in monocytes, $n=101$. (c) rs1981760 is remarkable for the profound differentiation this allele appears to have undergone in Asian populations with the derived $\mathrm{C}$ allele predominating in Asians (data from the 1000 genomes project $\left.{ }^{68}\right)$. rs1981760 and rs9302752 are in high linkage disequilibrium ( $r^{2}=0.98$ in our cohort). rs9302752-C is associated with leprosy risk in GWAS reports from Chinese populations 60 . The $C$ variant has a mild protective effect in CD independent of the deleterious 3020 ins $C$ frameshift mutation in NOD2 (ref. 59). (d) Quantitative PCR of NOD2 with two different primer sets performed blinded to genotype status in an independent set of 23 donors replicates the finding. Data representive of one primer-pair. (e) The rs1981760 SNP is located within a STAT3 transcription factor ChIP-seq binding site in MCF10A-Er-Src, an epithelial cell line (ENCODE). We investigated whether rs1981760 alters STAT3 binding in neutrophils by ChIP of STAT3 in four rs1981760 heterozygotes (screened to not have the 3020insC frameshift mutation in NOD2). Allele-specific probes in a digital droplet PCR assay demonstrate that the $C$ allele is significantly more frequently immunoprecipitated with STAT3 than the T-allele. $P$-values denote a $\chi^{2}$-test comparing expected versus actual ratio of positive and negative droplets for each donor. Tails show $95 \% \mathrm{Cl}$ of the enrichment estimate. (f) STAT3 expression levels differ between neutrophils and monocytes as measured by gene expression array, $n=101$. (g) After stimulation with muramyl dipeptide(MDP), the ligand for NOD2 and Pam3CSK4 (a co-stimulant) neutrophils from rs1981760-CC homozygous individuals express significantly higher levels of IFNB mRNA than TT homozygotes, and heterozygotes express intermediate levels (left panel). No such association is apparent in mock-treated control neutrophils (right panel). (b,d,f,g) Box lower and upper border denote 25th and 75th centiles, respectively, central line denotes median and whiskers extend to $1.5 \times 1$ QR.

\section{Methods}

Volunteer enrolment and cell purification. This study was approved by the Oxfordshire Research Ethics Committee (COREC reference 06/Q1605/55) and each individual gave informed consent to participation. The median age of the 101 included participants was 31 years (IQR 24-41), 50 were male and 51 female. For this substudy of our previously reported work ${ }^{19}$ we recruited 144 healthy Caucasian volunteers in Oxford, United Kingdom through the Oxford Biobank. A trained professional nurse and/or a medical doctor conducted a verbal review of clinical history to determine eligibility based on the absence of major chronic illness, current medication administration or symptoms of infection. We excluded 43 individuals because we did not have genotyping information at the time of this report ( 23 individuals) or the sample was an outlier after principal components analysis (20 individuals). We used an automated outlier detection algorithm 'detectOutlier' (lumi package) based on Euclidean distance to the centre of the cluster with iterative outlier removal and normalization. Whole blood was collected into sodium-heparin containing blood collection tubes (Becton Dickinson) and processed with $4-6 \mathrm{~h}$ after collection.

We isolated peripheral blood mononuclear cells by density-gradient centrifugation of blood-diluted with Hanks Buffered Saline solution (HBSS, Life Technologies, UK) layered on Lymphoprep (Axis-Shield, Norway) and sorted $\mathrm{CD} 14+$ monocytes using magnetic-activated cell sorting (MACS, Miltenyi Biotech) according to the manufacturer's instructions.

We isolated granulocytes using density-gradient centrifugation in which blood was layered on Polymorphrpep (Axis-Shield, Norway) according to the 
manufacturer's instructions. To remove red blood cell contamination we exposed granulocytes to endotoxin free cell-culture water (Life Technologies, UK) for $30 \mathrm{~s}$ and restored isosmosis using 2X HBSS. As neutrophils and eosinophils share density properties $\left(1.085 \mathrm{~g} \mathrm{ml}^{-1}\right)$, and previous studies demonstrated that ex vivo granulocyte transcriptomes are dominated by eosinophil transcripts despite their relative paucity ${ }^{27}$ ( $<5 \%$ of granulocytes), we further isolated $\mathrm{CD} 16+$ granulocytes as a pure population of neutrophils using CD16 + microbeads. Purity assessed on a representative sample was $>90 \%$. We performed full-blood counts on a Sysmex haematology analyser in a certified clinical laboratory; median neutrophil counts

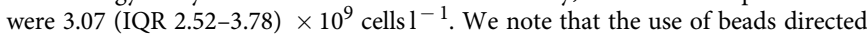
against CD16 may, in principle, alter or stimulate neutrophils although all purification steps were carried out on ice or at $4{ }^{\circ} \mathrm{C}$. Cell viability after isolation was $>80 \%$. Lysed cell pellets were immediately cryopreserved until extraction.

Genotyping. A total of 733,202 variants were genotyped using the Illumina HumanOmniExpress-12v1.0 Beadchip. QC steps included removal of individuals that were outliers by PCA, had poor genotyping call rates or heterozygosity measures. Variant QC included removal of SNP's with $\mathrm{MAF}<5 \%$, HWE $<1 \times 10^{-6}$ or poor call rates. Genome-wide imputation was performed by prephasing a scaffold of 588,170 SNP's with SHAPEIT ${ }^{66}$ and imputation in IMPUTE $2^{67}$ using the $1000 \mathrm{G}$ phase 1 release as the reference. Imputed SNP or indels with an info score $<0.9, \mathrm{MAF}<5 \%$ or departure from HWE $\left(1 \times 10^{-3}\right)$ were removed. In total $5,680,354$ variants were included in the eQTL analysis. Locations, where reported, are according to the human genome build GRCh37. Measures of $\mathrm{LD}$, where mentioned, are based on $1000 \mathrm{G} \mathrm{CEU}{ }^{68}$ or, if specifically mentioned, from this cohort of volunteers.

Gene expression analysis. During optimization of methods to isolate RNA from neutrophils we found that commonly used column-based isolation techniques were vastly outperformed by phenol-chloroform extraction using Trizol LS (Life Technologies) according to the manufacturer's instructions. This may be because a higher concentration of chaotropic and reducing agent is required to fully inhibit the abundance of nucleases in neutrophils. Therefore RNA was extracted using Trizol LS and further cleaned using the RNA MinElute cleanup kit (Qiagen, UK). RNA was quantified and integrity assessed using a Bioanalyser RNA 6000 Nano kit (Agilent, UK). Gene expression was quantified using the Illumina HumanHT-12 v4 BeadChip gene expression array platform with 47,231 probes according to the manufacturer's instructions. Samples were randomized across expression chips and run in a single batch.

Gene expression data were normalized using random-spline normalization, transformed by variance-stabilizing transformation and sample outliers were iteratively removed and normalization repeated. We note that of the 123 samples hybridized, 1 failed and 20 were removed as they were sample outliers-almost universally (19/20 samples) due to cRNA quality with these individuals having RNA size distribution $<800$ by BioAnalyser RNA 6000 Nano kit evaluation. We therefore did not obtain replacement arrays and hence all the data in this study derive from a single batch. Probe sequences mapping to more than one genomic location or regions with underlying polymorphisms frequent in $>1 \%$ of the population were excluded from eQTL analysis ( $n=1,8220$ probes). Only probes that were expressed and detected (GenomeStudio probe detection $P<0.01$ ) in neutrophils ( $n=11,023$ probes for 9,147 genes) were included in the primary analysis. For comparisons between neutrophils and monocytes, 10,012 probes for 8362 genes were included from 99 individuals.

eQTL mapping. We tested for association between genetic variation (using dosage estimates for imputed variants) within $1 \mathrm{Mb}$ of the expression probe (cis) or more distantly (trans), and gene expression in a linear regression framework implemented in 'MatrixEQTL'35 in $R$ (Matrix_eQTL_main function), incorporating principal components as covariates to account for hidden confounders (empirically determined number of PCs $=15$ ). We filtered results by a false discovery rate of $5 \%$ for cis associations or $1 \%$ for trans associations. We caution that this study, due to its modest size, is not adequately powered to exhaustively identify eQTL in neutrophils, or to map trans eQTL.

Annotation of eQTL with genomic features. Identified eQTL were annotated by location relative to the transcription start site or gene structures using data from the UCSC genome browser (GRCh37/b19) ${ }^{69}$.

We accessed whole-genome bisulfite sequencing and histone ChIP data generated by the BLUEPRINT consortium in primary human monocytes and neutrophils from 4 to 8 individuals (depending on feature). We defined features marked by an epigenetic mark as those present in at least half of all individuals (Supplementary Table 5).

For analyses of eQTL overlap with the location of epigenetic marks ${ }^{46}$, enhancers ${ }^{47,48}$, transcription factors ${ }^{49}$, conserved microRNA binding sites ${ }^{30}$ and in regions that have been under selection ${ }^{62}$, the location of the given feature relative to an eQTL was calculated using the 'GenomicRanges' package in $\mathrm{R}$ with the 'distanceToNearest' function. Fisher's exact test was used to evaluate enrichment relative to all imputed variants tested for eQTL (that is, those within $1 \mathrm{Mb}$ of a gene expressed in neutrophils and monocytes). We note that an implicit assumption in the Fisher's test is of independence of loci. The code used to generate the results is available from the authors.

Overlap between eQTL and trait-/disease-associated variants. All traits listed in the NHGRI Catalog GWAS (1138 traits as at 14 October 2014) were abstracted and expertly curated into one or more categories according to the disease system affected (28 potential categories) blind to GWAS or eSNP data. Groups were defined based on organ specificity of a particular trait, disease process or type. These included cardiovascular, respiratory, gastroenterological, urological, rheumatological, neurological, renal, endocrine, haematological, dermatological, bone, cancer, immunity and inflammation, autoimmune, allergy, genetic, viral infection, bacterial infection, parasitic disease, measurement, physiological, metabolic, chronic or degenerative disease, reproduction, drug related. Classification of a given trait was possible into multiple groups and to capture this diversity, for each trait we assigned trait membership into up to three possible groups.

A Fisher's exact test comparing the proportion of eQTL that are trait-associated variants or in linkage disequilibrium with these variants $\left(r^{2}>0.8\right.$ in 1000G Caucasian populations) with the proportion of all tested variants that are associated variants was performed stratified by disease category.

Neutrophil stimulation. To identify the role of rs1981760 in NOD2 downstream effects, we stimulated 5-10 $\times 10^{6}$ freshly isolated neutrophils resuspended at $2 \times 10^{6}$ cells per ml in RPMI1640 supplemented with $10 \%$ foetal calf serum and L-glutamine with $1 \mu \mathrm{g} \mathrm{ml}^{-1}$ muramyl dipeptide (n-acetyl) and $1 \mu \mathrm{g} / \mathrm{ml}$ Pam3CSK4, (both from Invivogen, UK) for two hours. Primer sequences for quantitative PCR are reported in Supplementary Data 13.

Chromatin immunoprecipitation. Crosslinked DNA from neutrophils was stored and subjected to ChIP after fragmentation of crosslinked DNA by sonication consisting two sets of nine cycles of $30 \mathrm{~s}$ each in a BioRuptor (Diagenode, Belgium) at high power. A mouse anti-human STAT3 antibody (Cell Signaling-NEB, UK, catalogue \#4904S) was used at a 1:50 dilution in conjunction with magnetic beads to immunoprecipitate STAT3 bound DNA. Quality control of ChIP-ped DNA was achieved by quantification on a Qubit 2.0 fluorometer (Invitrogen) using the Quant-iT dsDNA HS Assay Kit (Invitrogen).

Assessment of allele-specific STAT3 binding in neutrophils. For allele-specific digital droplet PCR quantification of rs1981760-C compared with rs1981760-T we designed allele-specific probes (Supplementary Data 13), and performed amplification using the digital droplet PCR Supermix for Probes (BioRad) according to the supplied protocol. The probe for the $\mathrm{T}$ and $\mathrm{C}$ allele were designed to fluoresce in different channels allowing simultaneous detection of both in a single reaction. Droplets were generated on a $\mathrm{q} \times 100$ droplet generator (BioRad, UK) and droplets read on a $\mathrm{q} \times 100$ droplet reader. Probes targeting SOCS3 and JAK3 were used as positive controls for ChIP of STAT3, and RAB4 as a negative control. Input DNA as well as DNA obtained after ChIP was amplified on the same plate. For allele-specific wells, we performed technical duplicates.

The proportion of positive droplets relative to negative droplets is associated with the absolute concentration of the product according to a Poisson distribution. We therefore exploited this to empirically calculate the expected proportion of $\mathrm{C}$ versus $\mathrm{T}$ droplets (so as to take into account possible probe efficiency differences) and compared this to the actual ratio after ChIP. $P$-values using a $\chi^{2}$-test with two degrees of freedom were used to estimate the probability of the observed versus the expected ratio being due to chance.

Software used for analysis. Analyses were conducted in $R$, using the following packages: limma; lumi; 'annotate'; 'vsn', 'GenomicRanges', 'qvalue', 'data.table'. For data presentation the 'gridExtra', 'ggbio', 'ggplot2' and 'VennDiagram' packages were used. Local association plots were created using LocusZoom. Ingenuity Pathway Analysis where used, was performed using a background set of all human genes on the Ilumina array. A listing of external files and their sources is given in Supplementary Data 14. FDR where shown denotes the false discovery rate calculated using the Benjamini Hochberg procedure using the p.adjust function.

\section{References}

1. Wright, F. A. et al. Heritability and genomics of gene expression in peripheral blood. Nat. Genet. 46, 430-437 (2014).

2. Wilhelm, M. et al. Mass-spectrometry-based draft of the human proteome. Nature 509, 582-587 (2014).

3. Dimas, A. S. et al. Common regulatory variation impacts gene expression in a cell type-dependent manner. Science 325, 1246-1250 (2009).

4. Lappalainen, T. et al. Transcriptome and genome sequencing uncovers functional variation in humans. Nature 501, 506-511 (2013).

5. Montgomery, S. B. et al. Transcriptome genetics using second generation sequencing in a Caucasian population. Nature 464, 773-777 (2010).

6. Pickrell, J. K. et al. Understanding mechanisms underlying human gene expression variation with RNA sequencing. Nature 464, 768-772 (2010). 
7. Stranger, B. E. et al. Patterns of cis regulatory variation in diverse human populations. PLoS Genet. 8, e1002639 (2012).

8. Stranger, B. E. et al. Population genomics of human gene expression. Nat. Genet. 39, 1217-1224 (2007).

9. Battle, A. et al. Characterizing the genetic basis of transcriptome diversity through RNA-sequencing of 922 individuals. Genome Res. 24, 14-24 (2014)

10. Fehrmann, R. S. et al. Trans-eQTLs reveal that independent genetic variants associated with a complex phenotype converge on intermediate genes, with a major role for the HLA. PLoS Genet. 7, e1002197 (2011).

11. Mehta, D. et al. Impact of common regulatory single-nucleotide variants on gene expression profiles in whole blood. Eur. J. Hum. Genet. 21, 48-54 (2013).

12. Westra, H. J. et al. Systematic identification of trans eQTLs as putative drivers of known disease associations. Nat. Genet. 45, 1238-1243 (2013).

13. Fairfax, B. P. et al. Genetics of gene expression in primary immune cells identifies cell type-specific master regulators and roles of HLA alleles. Nat. Genet. 44, 502-510 (2012)

14. Raj, T. et al. Polarization of the effects of autoimmune and neurodegenerative risk alleles in leukocytes. Science 344, 519-523 (2014).

15. Zeller, T. et al. Genetics and beyond--the transcriptome of human monocytes and disease susceptibility. PLOS ONE 5, e10693 (2010).

16. Lee, M. N. et al. Common genetic variants modulate pathogen-sensing responses in human dendritic cells. Science 343, 1246980 (2014).

17. Ferraro, A. et al. Interindividual variation in human T regulatory cells. Proc. Natl Acad. Sci. USA 111, E1111-E1120 (2014).

18. Barreiro, L. B. et al. Deciphering the genetic architecture of variation in the immune response to Mycobacterium tuberculosis infection. Proc. Natl Acad. Sci. USA 109, 1204-1209 (2012).

19. Fairfax, B. P. et al. Innate immune activity conditions the effect of regulatory variants upon monocyte gene expression. Science 343, 1246949 (2014).

20. Kim, S. et al. Characterizing the genetic basis of innate immune response in TLR4-activated human monocytes. Nat. Commun. 5, 5236 (2014).

21. Gibbs, J. R. et al. Abundant quantitative trait loci exist for DNA methylation and gene expression in human brain. PLoS Genet. 6, e1000952 (2010).

22. Nica, A. C. et al. The architecture of gene regulatory variation across multiple human tissues: the MuTHER study. PLoS Genet. 7, e1002003 (2011).

23. Ramasamy, A. et al. Genetic variability in the regulation of gene expression in ten regions of the human brain. Nat. Neurosci. 17, 1418-1428 (2014).

24. Schadt, E. E. et al. Mapping the genetic architecture of gene expression in human liver. PLoS Biol. 6, e107 (2008).

25. Mostafavi, S. et al. Variation and genetic control of gene expression in primary immunocytes across inbred mouse strains. J. Immunol. 193, 4485-4496 (2014).

26. Grisham, M. B., Engerson, T. D., McCord, J. M. \& Jones, H. P. A comparative study of neutrophil purification and function. J. Immunol. Methods 82, 315-320 (1985).

27. Stejskal, S., Koutna, I. \& Rucka, Z. Isolation of granulocytes: which transcriptome do we analyse-neutrophils or eosinophils? Folia. Biol. (Praha). 56, 252-255 (2010).

28. Westra, H. et al. Cell specific eQTL analysis without sorting cells. PLoS Genet. 11, e1005223 (2014).

29. Amulic, B., Cazalet, C., Hayes, G. L., Metzler, K. D. \& Zychlinsky, A. Neutrophil function: from mechanisms to disease. Annu. Rev. Immunol. 30, 459-489 (2012).

30. Kolaczkowska, E. \& Kubes, P. Neutrophil recruitment and function in health and inflammation. Nat. Rev. Immunol. 13, 159-175 (2013).

31. Bennouna, S., Bliss, S. K., Curiel, T. J. \& Denkers, E. Y. Cross-talk in the innate immune system: neutrophils instruct recruitment and activation of dendritic cells during microbial infection. J. Immunol. 171, 6052-6058 (2003).

32. Diana, J. et al. Crosstalk between neutrophils, B-1a cells and plasmacytoid dendritic cells initiates autoimmune diabetes. Nat. Med. 19, 65-73 (2013).

33. Pelletier, M. et al. Evidence for a cross-talk between human neutrophils and Th17 cells. Blood 115, 335-343 (2010).

34. von Bruhl, M. L. et al. Monocytes, neutrophils, and platelets cooperate to initiate and propagate venous thrombosis in mice in vivo. J. Exp. Med. 209, 819-835 (2012).

35. Shabalin, A. A. Matrix eQTL: ultra fast eQTL analysis via large matrix operations. Bioinformatics 28, 1353-1358 (2012).

36. Leek, J. T. \& Storey, J. D. Capturing heterogeneity in gene expression studies by surrogate variable analysis. PLoS Genet. 3, 1724-1735 (2007).

37. Buil, A. et al. C4BPB/C4BPA is a new susceptibility locus for venous thrombosis with unknown protein S-independent mechanism: results from genome-wide association and gene expression analyses followed by case-control studies. Blood 115, 4644-4650 (2010).

38. Neumann, K. et al. Clec12a is an inhibitory receptor for uric acid crystals that regulates inflammation in response to cell death. Immunity 40, 389-399 (2014).

39. Veyrieras, J. B. et al. High-resolution mapping of expression-QTLs yields insight into human gene regulation. PLoS Genet. 4, e1000214 (2008).

40. Dharmawardhane, S., Brownson, D., Lennartz, M. \& Bokoch, G. M. Localization of p21-activated kinase 1 (PAK1) to pseudopodia, membrane ruffles, and phagocytic cups in activated human neutrophils. J. Leukoc. Biol. 66, 521-527 (1999).
41. Bardoel, B. W., Kenny, E. F., Sollberger, G. \& Zychlinsky, A. The balancing act of neutrophils. Cell Host Microbe. 15, 526-536 (2014).

42. Borregaard, N., Sorensen, O. E. \& Theilgaard-Monch, K. Neutrophil granules: a library of innate immunity proteins. Trends Immunol. 28, 340-345 (2007).

43. McCracken, J. M. \& Allen, L. A. Regulation of human neutrophil apoptosis and lifespan in health and disease. J. Cell Death 7, 15-23 (2014).

44. Ambruso, D. R. et al. Human neutrophil immunodeficiency syndrome is associated with an inhibitory Rac2 mutation. Proc. Natl Acad. Sci. USA 97, 4654-4659 (2000).

45. Trynka, G. et al. Chromatin marks identify critical cell types for fine mapping complex trait variants. Nat. Genet. 45, 124-130 (2013).

46. Adams, D. et al. BLUEPRINT to decode the epigenetic signature written in blood. Nat. Biotechnol. 30, 224-226 (2012).

47. Andersson, R. et al. An atlas of active enhancers across human cell types and tissues. Nature 507, 455-461 (2014).

48. Consortium, F. et al. A promoter-level mammalian expression atlas. Nature 507, 462-470 (2014).

49. Boyle, A. P. et al. Annotation of functional variation in personal genomes using RegulomeDB. Genome Res. 22, 1790-1797 (2012).

50. Ravasi, T. et al. An atlas of combinatorial transcriptional regulation in mouse and man. Cell 140, 744-752 (2010).

51. Kim, D. H., Saetrom, P., Snove, Jr. O. \& Rossi, J. J. MicroRNA-directed transcriptional gene silencing in mammalian cells. Proc. Natl Acad. Sci. USA 105, 16230-16235 (2008)

52. Welter, D. et al. The NHGRI GWAS Catalog, a curated resource of SNP-trait associations. Nucleic Acids Res. 42, D1001-D1006 (2014).

53. Scott, D. L., Wolfe, F. \& Huizinga, T. W. Rheumatoid arthritis. Lancet 376, 1094-1108 (2010).

54. Okada, Y. et al. Genetics of rheumatoid arthritis contributes to biology and drug discovery. Nature 506, 376-381 (2014).

55. Khandpur, R. et al. NETs are a source of citrullinated autoantigens and stimulate inflammatory responses in rheumatoid arthritis. Sci. Transl. Med. 5, $178 \mathrm{ra1} 40$ (2013).

56. Sohn, D. H. et al.in American College of Rheumatology (Wiley, 2014).

57. Wright, H. L., Moots, R. J. \& Edwards, S. W. The multifactorial role of neutrophils in rheumatoid arthritis. Nat. Rev. Rheumatol. 10, 593-601 (2014).

58. Suzuki, A. et al. Functional haplotypes of PADI4, encoding citrullinating enzyme peptidylarginine deiminase 4 , are associated with rheumatoid arthritis Nat. Genet. 34, 395-402 (2003).

59. Jostins, L. et al. Host-microbe interactions have shaped the genetic architecture of inflammatory bowel disease. Nature 491, 119-124 (2012).

60. Zhang, F. R. et al. Genomewide association study of leprosy. N. Engl. J. Med. 361, 2609-2618 (2009).

61. Mayer-Barber, K. D. et al. Host-directed therapy of tuberculosis based on interleukin-1 and type I interferon crosstalk. Nature 511, 99-103 (2014).

62. Grossman, S. R. et al. Identifying recent adaptations in large-scale genomic data. Cell 152, 703-713 (2013).

63. Lionakis, M. S. et al. Chemokine receptor Ccr1 drives neutrophil-mediated kidney immunopathology and mortality in invasive candidiasis. PLoS. Pathog 8, e1002865 (2012).

64. Online Mendelian Inheritance in Man, OMIM. Johns Hopkins University, Baltimore, MD. MIM Number: 253220: 02/12/2014. http://omim.org/.

65. Sturm, R. A. et al. A single SNP in an evolutionary conserved region within intron 86 of the HERC2 gene determines human blue-brown eye colour. Am. J. Hum. Genet. 82, 424-431 (2008).

66. Delaneau, O., Marchini, J. \& Zagury, J. F. A linear complexity phasing method for thousands of genomes. Nat. Methods 9, 179-181 (2012).

67. Howie, B. N., Donnelly, P. \& Marchini, J. A flexible and accurate genotype imputation method for the next generation of genome-wide association studies. PLoS Genet. 5, e1000529 (2009).

68. Genomes Project, C. et al. An integrated map of genetic variation from 1,092 human genomes. Nature 491, 56-65 (2012).

69. Karolchik, D. et al. The UCSC Genome Browser database: 2014 update. Nucleic Acids Res. 42, D764-D770 (2014).

70. Betel, D., Koppal, A., Agius, P., Sander, C. \& Leslie, C. Comprehensive modeling of microRNA targets predicts functional non-conserved and noncanonical sites. Genome. Biol. 11, R90 (2010).

\section{Acknowledgements}

We thank the volunteers from the Oxford Biobank, NIHR Oxford Biomedical Research Centre, for their participation. The Oxford Biobank (www.oxfordbiobank.org.uk) is also part of the NIHR National Bioresource which supported the recalling process of the volunteers. This work was supported by the Wellcome Trust (Grants 074318 (J.C.K.), 088891 (B.P.F.), and 090532/Z/09/Z (core facilities Wellcome Trust Centre for Human Genetics including High-Throughput Genomics Group)), the European Research Council under the European Union's Seventh Framework Programme (FP7/2007-2013)/ 
ERC Grant agreement no. 281824 (J.C.K.), the Medical Research Council (98082 (J.C.K)) and the NIHR Oxford Biomedical Research Centre. V.N. was supported by the Rhodes Trust. We thank the volunteers for their participation in this study, Sr Jane Cheeseman for assistance in enrolling participants, Dr Katharine Plant for administrative assistance during the conduct of this study and Dr Christina Chang for help with illustrations. We also thank Prof's Yuta Kochi, Alison Simmons, Dr's Yukinori Okada and Luke Jostins and Mr Daniel Gaughan for helpful discussions.

\section{Author contributions}

V.N, B.P.F. and J.C.K. conceived, designed and initiated the study. V.N., B.P.F., J.C.K., and S.M. enrolled the volunteer cohort and performed sample collection. V.N., B.P.F. and D.W. performed the experimental work. V.N., B.P.F., P.H.,D.W., E.N. and J.C.K. Analysed the data. A.V.S.H. contributed reagents and expertise. V.N., B.P.F. and J.C.K. wrote the manuscript, with contributions to manuscript editing from other authors. All authors read and approved the manuscript before submission.

\section{Additional information}

Accession codes. Gene expression data is available through ArrayExpress

(E-MTAB-2232 \& E-MTAB-3536). Genotyping data have been deposited at the
European Genome- Phenome Archive (EGA) and is available on request to EGA (EGAS00000000109).

Supplementary Information accompanies this paper at http://www.nature.com/ naturecommunications

Competing financial interests: The authors declare no competing financial interests.

Reprints and permission information is available online at http://npg.nature.com/ reprintsandpermissions/

How to cite this article: Naranbhai, V. et al. Genomic modulators of gene expression in human neutrophils. Nat. Commun. 6:7545 doi: 10.1038/ncomms8545 (2015).

\section{(c) (1)}

This work is licensed under a Creative Commons Attribution 4.0 International License. The images or other third party material in this article are included in the article's Creative Commons license, unless indicated otherwise in the credit line; if the material is not included under the Creative Commons license, users will need to obtain permission from the license holder to reproduce the material. To view a copy of this license, visit http://creativecommons.org/licenses/by/4.0/ 